Pacific Journal of Mathematics

QUASI-COMPACTNESS AND DECOMPOSITIONS FOR 


\section{QUASI-COMPACTNESS AND DECOMPOSITIONS FOR ARBITRARY RELATIONS}

\section{STANLEY WeRtheimer}

If $T$ is a relation, $X$ the set of first elements and $Y$ a set containing all the second elements, $T(x)=\{y \in Y \mid(x, y) \in T\}$ and $T^{-1}(y)=\{x \in X \mid(x, y) \in T\}$. If $T(x) \cap T(y)$ is nonempty implies that $T(x)=T(y)$, the relation $T$ is semi-single-valued (ssv). Every $s s v$ surjection defines a decomposition of $X$ into point inverses and a decomposition of $Y$ into point images. G. T. Whyburn has analyzed the $s s v$ surjection $T$ on $X$ to $Y$ in terms of these decomposition spaces and the natural mappings onto these spaces. He discusses quasi-compactness for $s s v$ relations. It is the purpose of this paper to extend Whyburn's analysis to include all relations.

2. Decompositions. Let $P(X)$ denote the power set of $X$.

Definition 2.1. Let $T$ on $X$ to $Y$ be a relation. Define $\Delta T$ on $P(X)$ to $P(Y)$ by $\Delta T(A)=T(A) \cap T(X-A), \Delta^{*}$ the collection of nonempty subsets of $X$ for which $\Delta T(A)$ is empty, and $\Delta$ the collection of all minimal members of $\Delta^{*}$ with respect to the partial ordering defined by set inclusion.

The elements of $\Delta^{*}$ are the nonempty subsets $A$ of $X$ having the property that if $T^{-1}(y) \cap A$ is nonempty then $T^{-1}(y)$ is contained in $A$.

THEOREM 2.2. Let $T$ on $X$ to $Y$ be a relation, $I$ an indexing set and $A, A_{i}$ in $\Delta^{*}$ for all $i \in I$. Then (a) $X-A \in \Delta^{*}$ if $A$ is not $X$, (b) $T^{-1} T(A)=A$, (c) $T(X-A)=T(X)-T(A)$, (d) $\cap A_{i} \in \Delta^{*}$ if $\cap A_{i}$ is not empty, (e) $\bigcup A_{i} \in \Delta^{*}$, and (f) $A-A_{i} \in \Delta^{*}$ if $A-A_{i}$ is not empty.

Proof. (a) Since $A \in \Delta^{*}, \Delta T(X-A)=T(A) \cap T(X-A)=\Delta T(A)$ which is empty; thus $X-A \in \Delta^{*}$.

(b) Let $x \in T^{-1} T(A)$; then $T(x) \cap T(A)$ is not empty and, since $T(A) \cap T(X-A)=\varnothing$ (the empty set), $x \notin X-A$; i.e., $x \in A$. Thus $T^{-1} T(A) \subset A$. The reverse inclusion is always true, proving (b).

(c) Since $A \in \Delta^{*}$,

$$
\begin{aligned}
T(X-A) & =\left\{y \in Y \mid T^{-1}(y) \cap(X-A) \neq \varnothing\right\} \\
& =T(X)-\left\{y \in Y \mid T^{-1}(y) \subset A\right\}=T(X)-T(A) .
\end{aligned}
$$

(d) Suppose that $B=\bigcap A_{i} \notin \Delta^{*}$; then for some $x \in X-B, T(x) \cap$ $T(B) \neq \varnothing$. Also $x \in X-A_{j}$ for some $j \in I$ and so 


$$
T(x) \cap T(B) \subset T\left(X-A_{j}\right) \cap T\left(A_{j}\right)=\Delta T\left(A_{j}\right),
$$

since $T(B) \subset T\left(A_{i}\right)$ for all $i \in I$. This contradicts the fact that $A_{j} \in \Delta^{*}$.

(e) Let $C=\bigcup A_{i}$ and $y \in T(C)$. Then $y \in T\left(A_{k}\right)$ for some $k \in I$ and hence $y \notin T\left(X-A_{k}\right)$ since $\Delta T\left(A_{k}\right)=\varnothing$. Therefore $y \notin T(X-C)$, since $T(X-C) \subset T\left(X-A_{k}\right)$, and $\Delta T(C)=\varnothing$.

(f) Since both $A$ and $A_{i} \in \Delta^{*}$, so is $A \cap\left(X-A_{i}\right)=A-A_{i}$, from (d) and (a).

THEOREM 2.3. $\Delta$ is a decomposition of $X$.

Proof. For $A^{\prime}, A^{\prime \prime} \in \Delta^{*}$, if $A^{\prime} \cap A^{\prime \prime} \neq \varnothing, A^{\prime} \cap A^{\prime \prime} \in \Delta^{*}$ and also contained in $A^{\prime}$ and $A^{\prime \prime}$. By the minimality of $A^{\prime}$ and $A^{\prime \prime}, A^{\prime}=A^{\prime} \cap$ $A^{\prime \prime}=A^{\prime \prime}$. It is now necessary to show that $\mathrm{U} \Delta=X$. Let $x \in X$ and $A$ be the intersection of all members of $\Delta^{*}$ which contain $x$; then $A \in \Delta^{*}$ from (2.2) (d). Let $B \in \Delta^{*}$. If $x \in B, A \subset B$; if $x \in X-B, A \subset$ $X-B$ and $A \cap B=\varnothing$. Thus $A$ is minimal and hence in $\Delta$.

REMARK 2.4. If $T$ is $s s v$ the elements of $\Delta$ are point inverses, which are the members of the natural decomposition for such relations.

DEFINITION 2.5. $\Delta$ will be called the natural decomposition of $X$ induced by $T$. Let $X^{\prime}=\{D \in \Delta\}$ have the quotient topology and $P$ be the projection of $X$ onto $X^{\prime}$.

It is well known [4, p. 345] that the decomposition $\Delta$ is upper semicontinuous (usc) if and only if the mapping $P$ is closed. For the ssv case an equivalent condition for the decomposition to be usc is that $T$ be reflexive closed, i.e., $T^{-1} T(C)$ is closed for all closed sets $C$ in $X$ [2, p. 690]. This is not true for arbitrary relations, as is shown in the following example.

EXAMPLE 2.6. Let $\left\{x_{i}\right\}$ be any sequence of distinct real numbers converging to the real number $p$, and let $X=Y=\bigcup x_{i} \cup\{p\}$. Define $T$ on $X$ to $Y$ by $T\left(x_{i}\right)=\left\{x_{i}, x_{i+1}\right\}$ for all $i=1,2, \cdots$ and $T(p)=p$. Let $C$ be a closed subset of $X$; then $C$ is finite, or infinite containing $p$ and $T^{-1} T(C)$ is then finite, or infinite containing $p$ so that $T$ is reflexive closed. However, $\Delta=\left\{\bigcup x_{i},\{p\}\right\}$ and, for any $i, P^{-1} P\left(x_{i}\right)=$ $X-p$, which is not closed, so that $\Delta$ is not usc.

It will be shown that there is an analogous condition which reduces to that of being reflexive closed in the ssv case. Some preliminary ideas must be considered before stating the condition.

The relation $T^{-1}$ on $T(X)$ to $X$ defines sets corresponding to $\Delta^{*}$ and $\Delta$, say $\pi^{*}$ and $\pi$. It turns out, as one would expect by considering the $s s v$ case, that 
THEOREM 2.7. If $T$ on $X$ to $Z$ is a relation, then $\pi^{*}=T\left(\Delta^{*}\right)$ and $\pi=T(\Delta)$.

Proof. Let $Y=T(X)$. Since $U \Delta$ covers $X$ and $T$ on $X$ to $Y$ is a surjection, $U T(\Delta)$ covers $Y$. Now

$$
\begin{aligned}
\Delta T^{-1}(T(A)) & =T^{-1} T(A) \cap T^{-1}(Y-T(A))=A \cap\left(X-T^{-1} T(A)\right) \\
& =A \cap(X-A)=\varnothing
\end{aligned}
$$

for $A \in \Delta^{*}$, where the necessary algebra comes from (2.2); thus $T(A) \in$ $\pi^{*}$. Now let $\Delta T^{-1}(B)$ be empty and $A=T^{-1}(B)$; then $T(A) \cap T(X-$ $A)=T T^{-1}(B) \cap T\left(X-T^{-1}(B)\right)=B \cap(Y-B)=\varnothing$, so that $A \in \Delta^{*}$. Also $T(A)=T T^{-1}(B)=B$, and $T\left(\Delta^{*}\right)=\pi^{*}$.

Now let $B=T(A), A \in \Delta$; it will be shown that $B$ is minimal. Let $B^{\prime} \in \pi^{*}$. Then $B^{\prime}=T\left(A^{\prime}\right)$ for some $A^{\prime} \in \Delta^{*}$. If

$$
B \cap B^{\prime} \neq \varnothing, T(A) \cap T^{\prime}\left(A^{\prime}\right) \neq \varnothing .
$$

Let $y \in T(A) \cap T\left(A^{\prime}\right)$; then $T^{-1}(y) \subset A \cap A^{\prime}$ and, by the minimality of $A, A \subset A^{\prime}$ and $B=T(A) \subset T\left(A^{\prime}\right)=B^{\prime}$.

To see that any $B \in \pi$ is the image of something in $\Delta$, let $A=$ $T^{-1}(B)$, then $T(A)=T T^{-1}(B)=B$ from (2.2) (b) applied to $T^{-1}$. Since $B \in \pi^{*}, A \in \Delta^{*}$ from the previous paragraphs. Let $A^{\prime} \in \Delta^{*}$ and $A \cap$ $A^{\prime} \neq \varnothing$; then $T(A) \cap T\left(A^{\prime}\right) \neq \varnothing$ and, by the minimality of $T(A)$, $T(A) \subset T\left(A^{\prime}\right)$ and $A=T^{-1} T(A) \subset T^{-1} T\left(A^{\prime}\right)=A^{\prime}$. Therefore $A \in \Delta$, proving the theorem.

Definition 2.8. Let $T$ be a relation on $X$ to $Y$. For any $x \in X$, the order of $x, O(x)$, is the smallest positive integer $n$ so that $\left(T T^{-1}\right)^{n-1} T(x) \in \pi$. If there is no such integer then $O(x)$ is infinite.

The order of $T, O(T)$ is the smallest positive integer $n$ so that $O(x) \leqq n$ for all $x \in X$. If there is no such integer then the order of $T$ is infinite.

Theorem 2.9. Let $T$ on $X$ to $Y$ be a relation and $A$ a nonempty subset of $X$. Then $A$ is in $\Delta^{*}$ if and only if $T^{-1} T(A)=A$.

Proof. If $A \in \Delta^{*}, T^{-1} T(A)=A$, from (2.2)(b). Now suppose $T^{-1} T(A)=A$. Then $T^{-1} T(A) \cap(X-A)=A \cap(X-A)=\varnothing$; but

$$
T^{-1} T(A) \cap(X-A)=\varnothing
$$

if and only if $T(A) \cap T(X-A)=\varnothing$, and so $\Delta T(A)=\varnothing$; thus $A \in \Delta^{*}$.

Corollary 2.10. If $p$ is in $X$ and $B=\left(T T^{-1}\right)^{n-1} T(p), B=$ $T T^{-1}(B)$ if and only if $O(p) \leqq n$. 
An immediate consequence of the definition is that a relation $T$ is $s s v$ if and only if $O(T)=1$. The corollary to the next theorem gives a condition which ensures that the decomposition $\Delta$ is usc.

THEOREM 2.11. Let $T$ on $X$ to $Y$ be a relation and $O(T)=n$. Then $\Delta$ is usc if and only if $S^{n}=\left(T^{-1} T\right)^{n}$ is closed.

Proof. For any $x \in X,\left(T T^{-1}\right)^{n-1} T(x) \in \pi$ since $O(T)=n$ and thus, by (2.7), $S^{n}(x)=T^{-1}\left(T T^{-1}\right)^{n-1} T(x) \in \Delta$. Also, for any $A \in \Delta$ and subset $C$ of $X$ so that $A \cap C \neq \varnothing$, for $x \in A \cap C, A=S^{n}(x) \subset S^{n}(A \cap C) \subset$ $S^{n}(A)=A$; i.e., $A=S^{n}(A \cap C)=S^{n}(A)$. Therefore

$$
\begin{aligned}
S^{n}(C) & =S^{n}[\bigcup\{A \cap C \mid A \in \Delta, A \cap C \neq \varnothing\}] \\
& =\bigcup\left\{S^{n}(A \cap C) \mid A \in \Delta, A \cap C \neq \varnothing\right\} \\
& =\bigcup\{A \mid A \in \Delta, A \cap C \neq \varnothing\}=P^{-1} P(C),
\end{aligned}
$$

where $P$ is the projection mapping.

Thus, if $\Delta$ is usc, $P^{-1} P(C)$ is closed for $C$ closed and hence $S^{n}$ is closed. If $S^{n}$ is closed then $P^{-1} P(C)$ is closed for $C$ closed and $\Delta$ is usc.

Note that in Example (2.6), $O(T)$ is infinite since for any $x_{i}$ and any $n,\left(T T^{-1}\right)^{n-1} T\left(x_{i}\right)$ is not equal to $X-p$.

3. Quasi-compact relations. A ssv relation is quasi-compact if and only if the image of any closed inverse set is closed. This is equivalent to requiring that the image of any open inverse set be open for such relations, a situation which is not true in general for relations which are not $s s v$, as in shown by the next example.

EXAMPLE 3.1. Let $X=Y=[0,1]$ with the usual topology; let $p, q, r \in(0,1), p<q<r$. Define $T$ on $X$ to $Y$ by $T(x)=Y-q$ for $x \neq q$ and $T(q)=(p, r)$. For $q \in Y, T^{-1}(q)=q$, which is closed, while $T T^{-1}(q)=(p, r)$ which is not closed. The only open inverse sets are $X$ and $X-q$ and the image of both of these sets is open.

The next definition extends the concept of quasi-compactness to all relations in such a way as to be consistent with the definition for $s s v$ relations. In addition, all of Whyburn's results relating to decompositions for ssv relations [4] remain valid for arbitrary relations with the extended concept of a natural decomposition as discussed in the previous section.

Definition 3.2. The relation $T$ on $X$ to $Y$ is quasi-compact if and only if $T(A)$ is closed in $T(X)$ for each closed $A$ in $\Delta^{*}$. 
THEOREM 3.3. The relation $T$ is quasi-compact if and only if $T(A)$ is open in $T(X)$ for each open $A$ in $\Delta^{*}$.

Proof. Suppose $T$ is quasi-compact and $A$ (not $X$ ) in $\Delta^{*}$ is open; then $X-A \in \Delta^{*}$ by (2.2) (a) and $X-A$ is closed. Thus $T(X-A)=$ $T(X)-T(A)$ from (2.2) (c). Since $T(X-A)$ is closed in $T(X), T(A)$ is open in $T(X)$.

To prove the converse replace open with closed and closed with open in the above.

Definition 3.4. Let $T$ on $X$ to $Y$ be a relation, $x \in X$, and $A(x)$ the element in $\Delta$ containing $x$. The relation $t$ on $X$ to $Y$ is defined by $t(x)=T(A(x))$.

Note that $T(X)=t(X), T(A)=t(A)$ for $A$ in $\Delta$, and, if $T$ is $s s v$, $T=t$.

TheOREM 3.5. For any subset $A$ of $X, A$ is a member of $\Delta^{*}$ if and only if it is the union of the members of some subset of $\Delta$. In particular, if $A$ is in $\Delta^{*}, A$ is the union of all members of $\Delta$ which intersect $A$ non-vacuously.

Proof. Suppose $A \in \Delta^{*}$; then for each $x \in A, x \in A(x) \subset A$ by the minimality of $A(x)$. Therefore $A \subset \bigcup\{D \in \Delta \mid A \cap D \neq \varnothing\}$. The reverse inclusion is always true by the minimality condition. If $A$ is the union of the members of some subset of $\Delta, A \in \Delta^{*}$ by (2.2) (e).

Theorem 3.6. Let $T$ on $X$ to $Y$ be a relation. Then $t$ is ssv, and $T$ is quasi-compact if and only if $t$ is quasi-compact.

Proof. Let $x, x^{\prime} \in X$; then $t^{-1} t(x), t^{-1} t\left(x^{\prime}\right) \in \Delta$. Since $\Delta$ is a decomposition either $t(x)=t\left(x^{\prime}\right)$ or $t(x) \cap t\left(x^{\prime}\right)=\varnothing$; i.e., $t$ is $s s v$.

Since $t$ is $s s v$, the natural decomposition of $X$ induced by $t$ is $\delta=\left\{t^{-1} t(x) \mid x \in X\right\}$. To prove the second assertion it will be shown that $\delta$ coincides with $\Delta$ and hence, from (3.5), $\delta^{*}$ will equal $\Delta^{*}$. It will then be shown that $t(A)=T(A)$ for $A \in \Delta^{*}$ and thus for any closed $A \in \Delta^{*}$.

Note that for $p, q \in X$, if $T(A(p))=T(A(q))$ then

$$
A(p)=T^{-1} T(A(p))=T^{-1} T(A(q))=A(q) .
$$

Then

$$
\begin{aligned}
t^{-1} t(p) & =\{q \in X \mid t(p) \cap t(q) \neq \varnothing\}=\{q \in X \mid t(p)=t(q)\} \\
& =\{q \in X \mid T(A(p))=T(A(q))\}=\{q \in X \mid A(p)=A(q)\}=A(p),
\end{aligned}
$$


so $\delta=\Delta$.

Since $t(A)=T(A)$ for every $A \in \Delta$ and every member of $\Delta^{*}$ is the union of a subset of the elements of $\Delta, t\left(A^{\prime}\right)=T\left(A^{\prime}\right)$ for every $A^{\prime} \in \Delta^{*}$. This proves the assertion.

For two topological spaces, $X$ and $Y$, let $R(X, Y)$ be the collection of all relations on $X$ to $Y$ and $S(X, Y) \subset R(X, Y)$ be the set of all $s s v$ relations on $X$ to $Y$. Then $\rho$ on $R$ to $S$ defined by $\rho(T)=t$ is a surjection. The function $\rho$ thus defines an equivalence relation on the set of all relations given by $T \sim T^{\prime}$ if and only if $\rho(T)=\rho\left(T^{\prime}\right)$. From the last theorem either all of the members of any equivalence class are quasi-compact or they are all not quasi-compact, depending upon their representative in $S$. The function $\rho$ is itself quasi-compact since it is a retraction [4, Theorem (4.5), p. 346].

Now $P$ on $X$ to $X^{\prime}$ and $Q$ on $Y$ to $Y^{\prime}$ are the natural mappings for the decompositions of $X$ and $Y$ induced by the surjection $T$. Define $h$ on $X^{\prime}$ to $Y^{\prime}$ by $h(D)=Q t P^{-1}(D), g$ on $X$ to $Y^{\prime}$ by $g(x)=h P(x)$, and $s$ on $Y$ to $X^{\prime}$ by $s(y)=h^{-1} Q(y)$; then $P, Q, h, g$, and $s$ are onto functions, as pointed out by Whyburn [4]. The following theorem is a direct analogue of Theorem (6.1) in [4]; here the requirement that $T$ be $s s v$ is not needed. The proof is exactly the same since $T$ is quasi-compact if and only if $t$ is quasi-compact.

The following commutative diagram is useful for understanding the functions $h, g$, and $s$ and the theorem following.

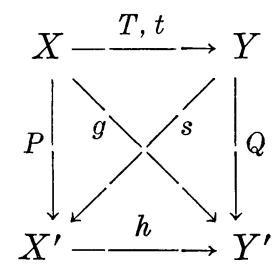

THEOREM 3.7. Let $T$ on $X$ to $Y$ be a surjection. Then

(a) $h$ is injective. Also $h\left(h^{-1}\right)$ is quasi-compact when $T\left(T^{-1}\right)$ is quasi-compact.

(b) $h$ is a homeomorphism if and only if $T$ and $T^{-1}$ are quasicompact.

(c) $g$ is quasi-compact when $T$ is quasi-compact and continusus when $T^{-1}$ is quasi-compact.

(d) $s$ is quasi-compact when $T^{-1}$ is quasi-compact and continuous when $T$ is quasi-compact.

The following theorem of Whyburn [4, Theorem (6.2), p. 348] analyzes the $s s v$ representative $t$ of $T$. A doubly quasi-compact relation is one for which both it and its inverse are quasi-compact. A 
relation is usc (lsc) if the inverse of every closed (open) set in its range is closed (open).

THEOREM 3.8. For any ssv doubly quasi-compact surjection $t$ on $X$ to $Y$ there is a topological space $Z$ and a pair of continuous quasicompact single-valued surjections $P$ on $X$ to $Z$ and $Q$ on $Y$ to $Z$ satisfying $t=Q^{-1} P$ and $t^{-1}=P^{-1} Q$. Thus $t$ is closed (open) if and only if $P$ is closed (open); equivalently, $t$ is usc (lsc) if and only if $Q$ is closed (open).

Conversely, any pair of quasi-compact single-valued continuous surjections $P$ on $X$ to $Z$ and $Q$ on $Y$ to $Z$ define a ssv doubly quasicompact relation under the definition $t(x)=Q^{-1} P(x), x$ in $X$, which in turn generate a pair $P^{\prime}$ and $Q^{\prime}$ equivalent to $P$ and $Q$.

Theorems (3.9), (3.10), and (3.11) are generalizations of Theorems (4.2), (4.4), and (4.5) in [4]. In every case Whyburn's results require that the relations be $s s v$.

THEOREM 3.9. Every usc compact-valued relation $T$ on $X$ to $Y$ is quasi-compact, for $X$ compact and $Y$ Hausdorff.

Proof. Let $C$ be closed in $X$; then $C$ is compact and $T(C)$ is compact since compactness is invariant under usc compact-valued relations [3, Corollary $\mathrm{A}_{2}$, p. 1497]. Since $Y$ is Hausdorff, $T(C)$ is closed and hence $T$ is closed and so quasi-compact.

For any surjection $T$ on $X$ to $Y$, a set $S \subset X$ is a cross-section for $T$ if $T(S)=Y$.

TheOREM 3.10. If $T$ on $X$ to $Y$ is a surjection so that $T \mid S$ is quasi-compact for some cross-section $S$ for $T$, then $T$ is quasi-compact.

Proof. Since $S$ is a cross-section for $T, S$ is a cross-section for $t$ since $T(S) \subset t(S)$. Thus $t$ is quasi-compact on $S$ by (3.6) and hence on $X$ by Whyburn's theorem. Again by (3.6), $T$ is quasi-compact on $X$.

A surjection $T$ on $X$ to $Y \subset X$ is retracting if $y$ is in $T(y)$ for each $y$ in $Y$.

TheOREM 3.11. Let $T$ on $X$ to $Y=X$ be retracting; then $T$ is quasi-compact.

Proof. If $T$ is retracting, $t$ is retracting since $T(y) \subset t(y)$ for every $y \in Y$. Thus $t$ is quasi-compact, from Whyburn, and hence $T$ is quasi-compact from (3.6). 
4. Local $\Delta$-connectedness. It is well known that every quotient space of a locally connected space is locally connected [1, Proposition 12, p. 112]. Whyburn [4] defines a space $Y$ to be locally connected relative to the surjection $T$ on $X$ to $Y$ provided that components of open image sets in $Y$ are open; he then proves that if $T$ is a $s s v$ doubly-quasi-compact surjection which preserves connectedness, $Y$ is locally connected relative to $T$ if $X$ is locally connected relative to $T^{-1}$. Since every quotient mapping is quasi-compact, this theorem contains the result in the first line of the section. It is the purpose of this section to extend the known results to arbitrary relations; in so doing, a theorem stronger than Whyburn's will be obtained for ssv relations.

Definition 4.1. Let $X$ be a space, $\Delta$ a decomposition of $X$, and $\Delta^{*}$ the collection of subsets of $X$ which are the union of the elements of some subset of $\Delta$. Then $X$ is locally $\Delta$-connected if the components of open $\Delta^{*}$ sets are open.

Lemma 4.2. Let $X$ be a topological space, $\triangle$ a decomposition of $X, X^{\prime}$ the quotient space of $X$ relative to $\Delta$, and $P$ the natural projection. If $D, D^{\prime}$ are subsets of $X^{\prime}$ so that $D$ is a component of $D^{\prime}$, then $P^{-1}(D)$ is the union of components of $P^{-1}\left(D^{\prime}\right)$.

Proof. It is sufficient to show that any component of $P^{-1}\left(D^{\prime}\right)$ intersecting $P^{-1}(D)$ is contained in $P^{-1}(D)$. Let $x \in P^{-1}(D)$ and $C$ be the component of $P^{-1}\left(D^{\prime}\right)$ containing $x$; then $P(C)$ is connected and hence $P(C) \subset D$ since $P(x) \in D$. Therefore $C \subset P^{-1} P(C) \subset P^{-1}(D)$.

Lemma 4.3. [1, Proposition 7, p. 110] Let $X$ be a topological space, $\triangle$ a decomposition of $X$, and $X^{\prime}$ the quotient space of $X$ relative to $\Delta$. If each element of $\Delta i$ is connected, then $X$ is connected when $X^{\prime}$ is connected.

The following theorem was suggested by Professor G. L. Cain, Jr.

THEOREm 4.4. Let $X$ be a topological space, $\Delta$ a decomposition of $X$ with connected elements, and $X^{\prime}$ the quotient space of $X$ relative to 4 . Then $X$ is locally -connected if and only if $X^{\prime}$ is locally connected.

Proof. Suppose that $X$ is locally $\Delta$-connected. Let $D^{\prime} \subset X^{\prime}$ be open and $D$ be a component of $D^{\prime}$. Since $D^{\prime}$ is open, $P^{-1}\left(D^{\prime}\right)$ is open; also, from (4.2), $P^{-1}(D)$ is the union of components of $P^{-1}\left(D^{\prime}\right)$, each of which is open since $X$ is locally $\Delta$-connected. Thus $P^{-1}(D)$ is open 
and hence $D$ is open, i.e., $X^{\prime}$ is locally connected.

Now suppose that $X^{\prime}$ is locally connected. Let $A^{\prime}$ be an open $\Delta^{*}$ set and $A$ a component of $A^{\prime}$. Since $P$ is continuous $P(A)$ is connected; let $B^{\prime}$ be the component of $P\left(A^{\prime}\right)$ containing $P(A)$.

Let the subspace $B=P^{-1}\left(B^{\prime}\right)$ of $X$ be decomposed by $\Delta$ restricted to $B$; then the subspace $B^{\prime}$ of $X^{\prime}$ is the quotient space of $B$ relative to $\triangle$ restricted to $B$. Since $B^{\prime}$ is connected, $B$ is connected, by (4.3). Also, since $P(A) \subset B^{\prime}, A \subset P^{-1} P(A) \subset P^{-1}\left(B^{\prime}\right)=B$. However, $A$ is a component of $A^{\prime}$ and $B$ is connected, so $B \subset A$; thus $A=B$.

Now $B^{\prime}$ is open since $X^{\prime}$ is locally connected, so $P^{-1}\left(B^{\prime}\right)=A$ is open; i.e., $X$ is locally $\Delta$-connected.

THEOREM 4.5. Let $T$ on $X$ to $Y$ be a doubly quasi-compact surjection, where $\pi$ has connected elements. If $X$ is locally $\Delta$-connected, $Y$ is locally $\pi$-connected.

Proof. The function $g$ in the diagram in $\S 3$ is a quasi-compact mapping, by (3.7). From Theorem (5.1) in [4], $Y^{\prime}$ is locally connected relative to $g$ since $g$ is a doubly quasi-compact function which preserves connectedness and $X$ is locally connected relative to $g^{-1}$, since $X$ is locally $\Delta$-connected. Since $g$ is single-valued, $Y^{\prime}$ is locally connected and hence, by (4.4), $Y$ is locally $\pi$-connected.

THeOREM 4.6. Let $T$ on $X$ to $Y$ be a relation. If $T$ has connected point images, $\pi$ has connected elements.

Proof. Let $G \in \pi$ and $G=A \cup B$, where $A \cap \operatorname{cl}(B)=B \cap \operatorname{cl}(A)=$ $\varnothing$. Now let $y \in T T^{-1}(A)$ and $x \in T^{-1}(y) \cap T^{-1}(A)$; then $T(x)$ is contained wholly in $A$ since $T(x)$ is connected and, since $y \in T(x), y \in A$; i.e., $T T^{-1}(A) \subset A$. The reverse inclusion is always true so that $T T^{-1}(A)=A$ and hence $A \in \pi$, by (2.9). But $A \subset G$, so $A=G$ and $B=\varnothing$, proving that $G$ is connected.

COROLlary 4.7. Let $T$ on $X$ to $Y$ be a doubly quasi-compact surjestion with connected point images. If $X$ is locally A-connected, $Y$ is locally $\pi$-connected.

REMARK 4.8. (4.7) includes Theorem (5.1) in [4]. Also included are the cases where $T$ has connected point images and is (open, $l s c$ ), (open, usc), (closed, lsc), or (closed, usc); in particular, the corollary includes the cases when $T$ is an open, closed, or quotient mapping. Since any locally connected space is locally $\Delta$-connected for any $\Delta$, the known results that local connectedness is preserved by open, closed, retracting, or quotient mappings are also included in (4.7). 
5. Reflexive closed and reflexive compact relations. A relation $T$ on $X$ to $Y$ is reflexive closed (compact) if $T^{-1} T(C)$ is closed (compact) for every closed (compact) subset $C$ of $X . X$ is a $k$-space if a subset $A$ of $X$ is closed when its intersection with every closed compact set in $X$ is closed. Theorems (5.1) and (5.5) are generalizations of Theorems 1, 2, 3, and 5 of E. Duda [2]. Duda's results are stated as corollaries to (5.1) and (5.5). Note that there are no continuity conditions in the hypotheses of the theorems while the functions in the corollaries are all assumed to be continuous.

Theorem 5.1. Let $X$ be a Hausdorff space and $T$ on $X$ to $Y$ a. relation so that $T^{-1} T(x)$ is compact for each $x$ in $X$. Then (a) implies (b); if $X$ is a k-space (b) implies (a); if $O(T)$ is finite (a) implies (c); if $X$ is a k-space and $O(T)$ is finite (b) implies (c); if $X$ is a k-space and $T$ is ssv, (a), (b), and (c) are equivalent.

(a) $T$ is reflexive closed.

(b) $T$ is reflexive compact.

(c) The natural decomposition of $X$ is usc.

Proof. (a) $\rightarrow(\mathrm{b})$ : Define $S$ on $X$ to $X$ by $S(x)=T^{-1} T(x)$. Then $x \in S(y)$ if and only if $T(x) \cap T(y) \neq \varnothing$ if and only if $y \in S(x)$; i.e., $S^{-1}(x)=\{y \in X \mid x \in S(y)\}=\{y \in X \mid y \in S(x)\}=S(x)$, and so any property of $S$ is also a property of $S^{-1}$. Since $T$ is reflexive closed, $S$ is closed and hence usc; $S$ has compact point images and therefore [3, Corollary $\mathrm{A}_{2}$, p. 1497] $S$ preserves compactness and is thus compact.

(b) $\rightarrow$ (a): The relation $S$ now preserves compactness and is thus compact; $S(x)$ is closed for all $x \in X$ and so from Corollary $\mathrm{C}_{5}^{\prime}$ on $\mathrm{p}$. 1499 of [3] which states that if $X$ and $Y$ are Hausdorff and $Y$ is a $k$-space, any compact relation with closed point values is closed, $S$ is closed.

(a) $\rightarrow$ (c): This follows from (2.12).

$(b) \rightarrow(c)$ : This follows from (b) $\rightarrow$ (a) and (a) $\rightarrow(c)$.

Corollary 5.2. Let $X$ be a Hausdorff $k$-space and $f$ a mapping of $X$ onto $Y$. If $f$ is reflexive compact then $f$ generates an upper semicontinuous decomposition.

COROLlARY 5.3. Let $f$ be a mapping with compact point inverses of a Hausdorff space $X$ onto a spase $Y$. If $f$ generates an usc decomposition then $f$ is reflexive compact.

COROLLARY 5.4. Let $f$ be a mapping with compact point inverses of a Hausdorff $k$-space into a space $Y$. The mapping $f$ generates an 
upper semicontinuous decomposition if and only if $f$ is reflexive compact.

A relation $T$ on $X$ to $Y$ is semi-closed if the image of every compact set in $X$ is closed in $Y$; thus, any continuous function onto a Hausdorff space is semi-closed with a semi-closed inverse.

THeOREM 5.5. If $X$ is a Hausdorff k-space and $T$ on $X$ to $Y$ is a quasi-compact reflexive compact surjection of finite order with $T^{-1}$ semi-closed, then $T$ is compact.

Proof. All relations referred to are those in the diagram preceding (3.7). Since $T$ is reflexive compact, the decomposition $\Delta$ of $X$ is usc, by (5.1). From (3.7) (d), $s$ is continuous since $T$ is quasicompact, and thus $s$ preserves compactness.

For $A \in X^{\prime}, x \in A, P^{-1}(x)=\left(T^{-1} T\right)^{n}(x)$ for some $n$ since $O(T)$ is finite; since $T$ is reflexive compact $P$ has compact point inverses. Moreover $\Delta$ is usc and so $P$ is closed and hence compact [3, Corollary $\mathrm{A}_{3}$, p. 1497]. Thus $t^{-1}(K)$ is compact for any compact subset $K$ of $Y$ since $t^{-1}=P^{-1} s$.

Since $T^{-1}$ is semi-closed, $T^{-1}(K)$ is closed; therefore $T^{-1}(K)$ is a closed subset of the compact set $t^{-1}(K)$ and is thus compact. This proves the theorem.

CoRollary 5.6. Let $f$ on $X$ to $Y$ be an onto mapping, where $X$ is a Hausdorff $k$-space. If $f$ is quasi-compact and reflexive compact, then $f$ is compact.

\section{REFERENCES}

1. N. Bourbaki, General topology-Part 1, Addison-Wesley, Reading, Mass. 1966.

2. E. Duda, Reflexive compact mappings, Proc. Amer. Math. Soc., 17 (1966), 688-693.

3. G. T. Whyburn, Continuity of multifunctions, Proc. Nat. Acad. Sci., U.S.A., 54 (1965), 1494-1501.

4. —, Retracting multifunctions, Proc. Nat. Acad. Sci., U.S.A., 59 (1968), 343348.

Received June 2, 1970. Portions of this paper are from Chapter 3 of the author's doctoral thesis. This work was carried out while the author was a National Science Foundation Science Faculty Fellow.

Georgia Institute of TeChNology 



\section{PACIFIC JOURNAL OF MATHEMATICS}

\section{EDITORS}

H. SAMELSON

Stanford University

Stanford, California 94305

C. R. Новву

University of Washington

Seattle, Washington 98105
J. DUGundJI

Department of Mathematics

University of Southern California

Los Angeles, California 90007

RICHARD ARENS

University of California

Los Angeles, California 90024

\section{ASSOCIATE EDITORS}
E. F. BECKENBACH
B. H. NeumanN
F. WOLF
K. YOSHIDA

\section{SUPPORTING INSTITUTIONS}
UNIVERSITY OF BRITISH COLUMBIA
CALIFORNIA INSTITUTE OF TECHNOLOGY
UNIVERSITY OF CALIFORNIA
MONTANA STATE UNIVERSITY
UNIVERSITY OF NEVADA
NEW MEXICO STATE UNIVERSITY
OREGON STATE UNIVERSITY
UNIVERSITY OF OREGON
OSAKA UNIVERSITY
UNIVERSITY OF SOUTHERN CALIFORNIA
STANFORD UNIVERSITY
UNIVERSITY OF TOKYO
UNIVERSITY OF UTAH
WASHINGTON STATE UNIVERSITY
UNIVERSITY OF WASHINGTON
AMERICAN MATHEMATICAL SOCIETY CHEVRON RESEARCH CORPORATION TRW SYSTEMS
NAVAL WEAPONS CENTER 


\section{Pacific Journal of Mathematics}

\section{Vol. 37, No. $1 \quad$ January, 1971}

Gregory Frank Bachelis and Haskell Paul Rosenthal, On unconditionally

converging series and biorthogonal systems in a Banach space .........

Richard William Beals, On spectral theory and scattering for elliptic

operators with singular potentials .........................

J. Lennart (John) Berggren, Solvable and supersolvable groups in which every element is conjugate to its inverse ........................ 21

Lindsay Nathan Childs, On covering spaces and Galois extensions ..........

William Jay Davis, David William Dean and Ivan Singer, Multipliers and

unconditional convergence of biorthogonal expansions..............

Leroy John Derr, Triangular matrices with the isoclinal property ............

Paul Erdős, Robert James McEliece and Herbert Taylor, Ramsey bounds for

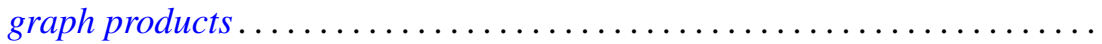

Edward Graham Evans, Jr., On epimorphisms to finitely generated

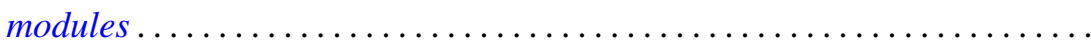

Hector O. Fattorini, The abstract Goursat problem ................. 51

Robert Dutton Fray and David Paul Roselle, Weighted lattice paths .........

Thomas L. Goulding and Augusto H. Ortiz, Structure of semiprime $(p, q)$

radicals ...........................................

E. W. Johnson and J. P. Lediaev, Structure of Noether lattices with join-principal maximal elements ....

David Samuel Kinderlehrer, The regularity of minimal surfaces defined over

slit domains

Alistair H. Lachlan, The transcendental rank of a theory. .

Frank David Lesley, Differentiability of minimal surfaces at the boundary ...

Wolfgang Liebert, Characterization of the endomorphism rings of divisible torsion modules and reduced complete torsion-free modules over complete discrete valuation rings....

Lawrence Carlton Moore, Strictly increasing Riesz norms.

Raymond Moos Redheffer, An inequality for the Hilbert transform ...

James Ted Rogers Jr., Mapping solenoids onto strongly self-entwined,

circle-like continua..........................

Sherman K. Stein, B-sets and planar maps ................... 217

Darrell R. Turnidge, Torsion theories and rings of quotients of Morita

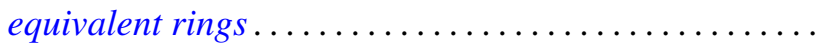

Fred Ustina, The Hausdorff means of double Fourier series and the principle of localization ................................

Stanley Joseph Wertheimer, Quasi-compactness and decompositions for arbitrary relations.

Howard Henry Wicke and John Mays Worrell Jr., On the open continuous images of paracompact $\check{C}$ ech complete spaces... 